\title{
Integrated Design Of An Observer-based Fault Detection System Over Unreliable Digital Channels
}

\author{
W. Li and S. X. Ding
}

\begin{abstract}
In this paper, the observer-based fault detection problem over an unreliable digital channel is studied. The channel qualities, which are usually called Quality of Service $(\mathrm{QoS})$, i.e. packet loss probability and quantization error, are first analyzed in the view of control engineering, and they are transferred into stochastic parameters and system uncertainties. Then it is shown that the fault detection system can be modeled in a framework of stochastic uncertain systems. With a given observer the requirements of QoS for an expected fault detection performance are established by solving an optimization problem with the help of linear matrix inequalities (LMIs). Then for the given QoS of a channel, the optimal observer for fault detection is also derived. Finally the integrated design of QoS and the fault detection system is discussed.
\end{abstract}

\section{INTRODUCTION}

In last decades, networked control systems (NCS) have received more and more attentions due to their promising applications in industrial processes and mechatronic systems (robotics, automotive and etc). By using networks, large and complex implementation as well as the modularity and reconfigurability can be realized in the control system. In fact NCS consists of two subsystems: the control part and the communication part. The modern communication channels are usually digital and unreliable. It may encounter packet losses and quantization errors due to disturbances from the environment or limitations of the channel itself. Those unreliabilities are used to represent the QoS of the digital channel, and they can significantly influence the performance of control systems. Therefore on one hand the design of NCS must take QoS into account and in the other hand the digital network should be designed to provide proper QoS in order to guarantee the performance of NCS. An integrated design of NCS considering the control part and the communication part is also of interests.

There are many contributions have been published about how to deal with the unreliabilities of the system. In [7] [8] [10] [11] [12] [14] and [16], packet loss was intensively analyzed. The optimal control with packet losses was studied in [7] by applying dynamic programming technique. In [8] and [11] the state estimation problems with packet loss were solved with the help of Kalman filtering theory. In [10]

This work was supported in part by the German Research Foundation (DFG).

W. $\mathrm{Li}$ is with the Institute of Automatic Control and Complex Systems, University of Duisburg-Essen, Duisburg, 47057, Germany phone: +492033794293; fax: +492033792928; e-mail: wei.li@uni-due.de

S. X. Ding is with the Institute of Automatic Control and Complex Systems, University of Duisburg-Essen, Duisburg, 47057, Germany e-mai I : steven.ding@uni-due.de
[12] and [14] the systems with packet losses were modeled as Markov jumping systems and the so-called stochastic stability of the system can be achieved. The fault detection system considering packet losses was designed in [16] by using Markov jumping system theory. There are also many works discussing the quantized feedback control systems. The coarsest memoryless static quantizer [1] [2] [5] [6] and dynamic quantizer [9] were designed which guarantee the system stability. Those works indicate that the feedback information can be useful with different levels of resolutions for different levels of system performances. In order to stabilize the system the minimum feedback information must be enough to compensate for the increase in the uncertainty due to the quantization [5]. Those contributions are mainly concentrated on dealing with QoS, rather than integrated design of control and communication parts of NCS. Besides, [3] and [4] studied the fault detection problem of stochastic systems.

In this paper an observer-based fault detection system over unreliable digital channels is designed by applying a framework of stochastic uncertain systems. The stochastic uncertain system can describe deterministic uncertainties and also stochastic uncertainties. We first study QoS, including packet losses and quantization errors, of digital channels and reformulate them in terms of stochastic variables and system uncertainties. An observer is proposed to estimate the system outputs and generate residual signals. Such an observer is also called fault detection filter (FDF). Residual signals are evaluated and compared with a threshold to test the occurrences of system faults. The process, FDF and the digital channel are then modeled as a whole system in the framework of the stochastic uncertain system. Based on that, an integrated design of FDF and communication channels is proposed.

This paper is organized as follows. In section II, we formulated the fault detection problem over unreliable digital channels and analyze the characteristics of the channels. In section III, preliminaries of the framework of stochastic uncertain systems are introduced and then the design approach of the FDF and digital channels is presented. In section IV, a numerical example is given to illustrate the results. Finally, a conclusion is given in section $\mathrm{V}$.

\section{PROBLEM FORMULATION}

The communication channel always encounter unreliabilities, e.g. packet loss and quantization error. Fig. 1 gives the structure of the system under consideration. The measurement signals first go through source encoder where the sig- 
nals are transferred into a binary sequence after quantization. Then it is transmitted via the channel where the transmitted packets can be lost. The process is a linear time invariant discrete system

$$
\begin{aligned}
x(k+1) & =A x(k)+B u(k)+E_{d} d(k)+E_{f} f(k) \\
y(k) & =C x(k)+F_{d} d(k)+F_{f} f(k) \\
u(z) & =K(z) y(z)
\end{aligned}
$$

where $x \in R^{n}$ is the state vector, $y \in R^{m}$ is the measurements, $u \in R^{p}$ is the control inputs, $d \in R^{n_{d}}$ is the disturbance vector, and $f \in R^{n_{f}}$ is the fault vector. $A, B, C, E_{d}, E_{f}, F_{d}, F_{f}$ are known matrices with compatible dimensions. $K(z)$ stands for the output feedback controller applied in the process, which can be a static or dynamic one. Let

$$
x_{c l}(k)=\left[\begin{array}{c}
x(k) \\
x_{c}(k)
\end{array}\right]
$$

where $x_{c}(k)$ is the state of the output feedback controller. Then the close-loop system of (1) can be written as

$$
\begin{aligned}
x_{c l}(k+1) & =A_{c l} x(k)+E_{d, c l} d(k)+E_{f, c l} f(k) \\
y(k) & =C_{c l} x_{c l}(k)+F_{d, c l} d(k)+F_{f, c l} f(k)
\end{aligned}
$$

The fault detection (FD) system receives the transmitted

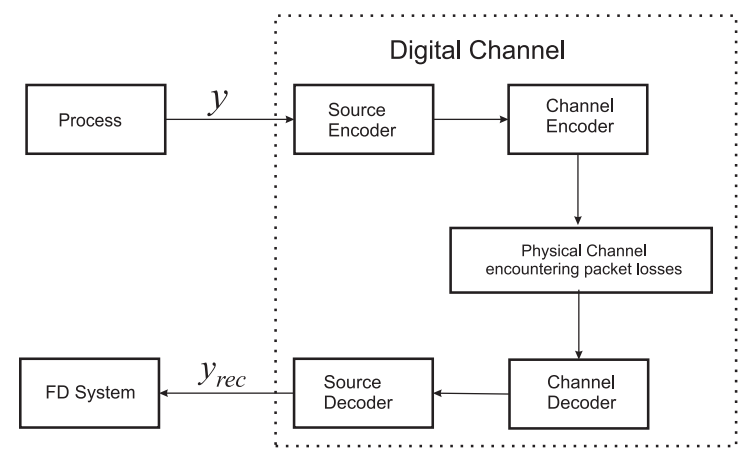

Fig. 1. Remote Fault Detection System

measurements after source decoding at the remote side, and then it generates the residual signals. The controller is located at the process side. The control law is assumed to be known to the fault detection system. In this fault detection system only the process measurements are transmitted over the digital channel, and therefore $u$ is not directly available at the remote side. Hence we suggest the following FDF for the closed-loop system (2) with deterministic disturbances

$$
\begin{aligned}
\hat{x}_{c l}(k+1) & =A_{c l} \hat{x}(k)+L\left(y_{r e c}(k)-\hat{y}(k)\right) \\
\hat{y}(k) & =C_{c l} \hat{x}(k) \\
r(k) & =y_{r e c}(k)-\hat{y}(k)
\end{aligned}
$$

where $r \in R^{m}$ is the residual signal. $y_{\text {rec }}$ is the received measurements at the remote side. $L$ is the observer gain which should be designed to ensure the stability and the dynamics of the FDF. From the viewpoint of residual generation, it may be of additional advantage to adopt the closed-loop FD, e.g. using a decoupling controller will reduce an MIMO problem to a number of SISO problem.
The unreliabilities of networks considered in this paper include packet losses and quantization errors. First the measurements can be lost during the transmission and we assume its probability is $P_{l}$. Second we assume that the source encoder applies the following logarithmic quantizer suggested by [2]

$$
Q(v)= \begin{cases}\rho^{i} v_{0} & \text { if } \frac{1}{1+\delta_{q}} \rho^{i} v_{0}<v \leq \frac{1}{1-\delta_{q}} \rho^{i} v_{0}, v>0 \\ 0 & \text { if } v=0 \\ -Q(-v) & \text { if } v<0\end{cases}
$$

where $0<\rho<1$ is the quantization density, $v_{0}>0$ is the maximum possible value of $v$, and

$$
\delta_{q}=\frac{1-\rho}{1+\rho}
$$

From [2], it is known that a suitable model for the logarithmic quantizer $Q(v)$ with parameter $\delta_{q}$ consists in the following multiplicative random map

$$
Q(v)=\left(1+\Delta_{q}\right) v
$$

where $\Delta_{q} \in\left[-\delta_{q}, \delta_{q}\right]$. Hence $\delta_{q}$ stands for the bound of the uncertainty introduced by the quantizer.

It is clear that the QoS of the considered channel can be described with $P_{l}$ and $\delta_{q}$.

\section{Fault Detection System Design}

The fault detection system should be designed to minimize the influence of disturbance so that an occurrence of faults can be detected earlier. In this section, we first give a framework of stochastic uncertain systems. Then we analyze and design the fault detection system with packet losses and quantization errors by applying the framework.

\section{A. A framework of stochastic uncertain systems}

In order to design the fault detection system over communication channels, we first introduce the following stochastic uncertain system

$$
\begin{aligned}
x(k+1) & =\left(A_{0}+\Delta A\right) x(k)+\left(E_{w, 0}+\Delta E_{w}\right) w(k) \\
r(k) & =\left(C_{0}+\Delta C\right) x(k)+\left(F_{w, 0}+\Delta F_{w}\right) w(k)
\end{aligned}
$$

where $x \in \mathbf{R}^{n}$ denotes the state vector, $r \in \mathbf{R}^{m}$ denotes the output vector and $w \in \mathbf{R}^{n_{w}}$ denotes the inputs of the system. $A_{0}, C_{0}, E_{w, 0}$ and $F_{w, 0}$ are known real matrices of compatible dimension and

$$
\begin{aligned}
& {\left[\begin{array}{cc}
\Delta A & \Delta E_{w} \\
\Delta C & \Delta F_{w}
\end{array}\right]=\left[\begin{array}{cc}
\Delta A_{0} & \Delta E_{w, 0} \\
\Delta C_{0} & \Delta F_{w, 0}
\end{array}\right]} \\
& +\sum_{i=1}^{l}\left[\begin{array}{cc}
A_{i}+\Delta A_{i} & E_{w, i}+\Delta E_{w, i} \\
C_{i}+\Delta C_{i} & F_{w, i}+\Delta F_{w, i}
\end{array}\right] p_{i}
\end{aligned}
$$

where

$$
\left[\begin{array}{cc}
\Delta A_{j} & \Delta E_{w, j} \\
\Delta C_{j} & \Delta F_{w, j}
\end{array}\right]=\left[\begin{array}{c}
E_{j} \\
F_{j}
\end{array}\right] \Delta\left[\begin{array}{ll}
G & J
\end{array}\right]
$$

with known matrices $A_{j}, C_{j}, E_{w, j}, F_{w, j}$ and $E_{j}, F_{j}, G, J$, $j=0, \cdots, l$ of appropriate dimensions and

$$
\Delta \Delta^{T} \leq I
$$


Here $p^{T}(k)=\left[p_{1}(k) \cdots p_{l}(k)\right]$ represents the stochastic model uncertainties with

$$
\bar{p}(k)=E(p(k))=0, E\left(p(k) p(k)^{T}\right)=\operatorname{diag}\left(\sigma_{1}^{2}, \cdots, \sigma_{l}^{2}\right)
$$

where $\sigma_{i}, i=1, \cdots, l$ are known. It is further assumed that $p(0), p(1), \cdots$, are independent.

For the system we give the following theorem.

Theorem 1: Given system (4) and a constant $\mu>0$, then

$$
\|r\|_{2}=\sqrt{\sum_{0}^{\infty} E\left(r(k)^{T} r(k)\right)}<\mu\|w\|_{2},
$$

if the following linear matrix equality holds for some $Y>0$ and $\varepsilon>0$

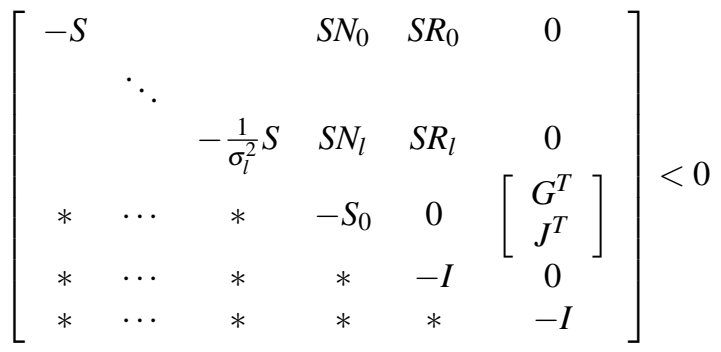

with

$$
\begin{aligned}
N_{j} & =\left[\begin{array}{ll}
A_{j} & E_{j} \\
C_{j} & F_{j}
\end{array}\right], R_{j}=\left[\begin{array}{c}
E_{j} \\
F_{j}
\end{array}\right], j=0, \cdots, l, \\
S & =\left[\begin{array}{cc}
Y & 0 \\
0 & \varepsilon I
\end{array}\right], S_{0}=\left[\begin{array}{cc}
Y & 0 \\
0 & \mu^{2} \varepsilon I
\end{array}\right] .
\end{aligned}
$$

Proof: See Appendix.

This linear matrix inequality can be solved via existing efficient numerical methods.

\section{B. Residual Dynamics}

Define $e(k)=x_{c l}(k)-\hat{x}_{c l}(k)$, and then we can get the following augmented system if there is no packet loss:

$$
\begin{aligned}
{\left[\begin{array}{c}
x_{c l}(k+1) \\
e(k+1)
\end{array}\right]=} & {\left[\begin{array}{cc}
A_{c l} & 0 \\
-L \Delta_{q} C_{c l} & A_{c l}-L C_{c l}
\end{array}\right]\left[\begin{array}{c}
x_{c l}(k) \\
e(k)
\end{array}\right] } \\
& +\left[\begin{array}{c}
E_{d, c l} \\
E_{d, c l}-L F_{d, c l}-L \Delta_{q} F_{d, c l} \\
E_{f, c l} \\
E_{f, c l}-L F_{f, c l}-L \Delta_{q} F_{f, c l}
\end{array}\right] d(k)
\end{aligned}
$$

and

$$
\begin{aligned}
r(k)= & {\left[\begin{array}{ll}
\Delta_{q} C_{c l} & C_{c l}
\end{array}\right]\left[\begin{array}{c}
x(k) \\
e(k)
\end{array}\right]+\left(F_{d, c l}+\Delta_{q} F_{d, c l}\right) d(k) } \\
& +\left(F_{f, c l}+\Delta_{q} F_{f, c l}\right) f(k)
\end{aligned}
$$

If a packet loss occurs at the time instant $k$, we apply $y_{\text {rec }}(k)=\hat{y}(k)$. Then the residual dynamics is governed by

$$
\begin{aligned}
{\left[\begin{array}{c}
x_{c l}(k+1) \\
e(k+1)
\end{array}\right]=} & {\left[\begin{array}{cc}
A_{c l} & 0 \\
0 & A_{c l}
\end{array}\right]\left[\begin{array}{c}
x_{c l}(k) \\
e(k)
\end{array}\right] } \\
& +\left[\begin{array}{c}
E_{d, c l} \\
E_{d, c l}
\end{array}\right] d(k)+\left[\begin{array}{c}
E_{f, c l} \\
E_{f, c l}
\end{array}\right] f(k)
\end{aligned}
$$

and

$$
r(k)=\left[\begin{array}{ll}
0 & 0
\end{array}\right]\left[\begin{array}{c}
x_{c l}(k) \\
e(k)
\end{array}\right]
$$

We assume that the packet loss is independent and identically distributed with probability $P_{l}$. Then in fault-free case, i.e. $f=0$, the above system can be reformulated in the manner of (4) with

$$
\begin{aligned}
A_{0} & =\left[\begin{array}{cc}
A_{c l} & 0 \\
0 & A_{c l}-\left(1-P_{l}\right) L C_{c l}
\end{array}\right], \\
A_{1} & =\left[\begin{array}{cc}
0 & 0 \\
0 & -L C_{c l}
\end{array}\right], \\
\Delta A_{0} & =\left[\begin{array}{cc}
0 & 0 \\
\left(1-P_{l}\right) \delta_{q} L \Delta C_{c l} & 0
\end{array}\right], \Delta A_{1}=\left[\begin{array}{cc}
0 & 0 \\
\delta_{q} L \Delta C_{c l} & 0
\end{array}\right], \\
C_{0} & =\left[\begin{array}{cc}
0 & \left(1-P_{l}\right) C_{c l}
\end{array}\right], C_{1}=\left[\begin{array}{ll}
0 & C_{c l}
\end{array}\right], \\
\Delta C_{0} & =\left[\begin{array}{ll}
\left(1-P_{l}\right) \delta_{q} \Delta C_{c l} & 0
\end{array}\right], \Delta C_{1}=\left[\begin{array}{ll}
\delta_{q} \Delta C_{c l} & 0
\end{array}\right]
\end{aligned}
$$

and for the disturbance inputs $d$,

$$
\begin{aligned}
E_{d, 0}= & {\left[\begin{array}{c}
E_{d, c l} \\
E_{d, c l}-\left(1-P_{l}\right) L F_{d, c l}
\end{array}\right], } \\
E_{d, 1}= & {\left[\begin{array}{c}
0 \\
-L F_{d, c l}
\end{array}\right], } \\
\Delta E_{d, 0}= & {\left[\begin{array}{c}
0 \\
\left(1-P_{l}\right) \delta_{q} L \Delta F_{d, c l}
\end{array}\right], \Delta E_{d, 1}=\left[\begin{array}{c}
0 \\
\delta_{q} L \Delta F_{d, c l}
\end{array}\right] } \\
F_{d, 0}= & \left(1-P_{l}\right) F_{d, c l}, F_{d, 1}=F_{d, c l}, \\
\Delta F_{d, 0}= & \left(1-P_{l}\right) \delta_{q} \Delta F_{d, c l}, \Delta F_{d, 1}=\delta_{q} \Delta F_{d, c l}
\end{aligned}
$$

and

$$
\begin{aligned}
& p_{1}=P_{l}, \text { if there is no packet loss } \\
& p_{1}=-\left(1-P_{l}\right), \text { if there is packet loss } \\
& \sigma_{1}^{2}=\left(1-P_{l}\right) P_{l}
\end{aligned}
$$

where $\Delta \Delta^{T} \leq I$. Those uncertainties can be written as

$$
\left[\begin{array}{cc}
\Delta A_{j} & \Delta E_{d, j} \\
\Delta C_{j} & \Delta F_{d, j}
\end{array}\right]=\left[\begin{array}{c}
E_{j} \\
F_{j}
\end{array}\right] \Delta\left[\begin{array}{ll}
G & J
\end{array}\right], j=0,1
$$

with

$$
\begin{aligned}
E_{0} & =\left[\begin{array}{cc}
0 & 0 \\
\left(1-P_{l}\right) \delta_{q} L & 0
\end{array}\right], E_{1}=\left[\begin{array}{cc}
0 & 0 \\
\delta_{q} L & 0
\end{array}\right], \\
F_{0} & =\left[\begin{array}{ll}
\left(1-P_{l}\right) \delta_{q} & 0
\end{array}\right], F_{1}=\left[\begin{array}{ll}
\delta_{q} & 0
\end{array}\right], \\
G & =\left[\begin{array}{ll}
C_{c l} & 0
\end{array}\right], J=\left[\begin{array}{c}
F_{d, c l} \\
0
\end{array}\right] .
\end{aligned}
$$

It is clear that, the whole system is characterized with the QoS parameters $P_{l}, \delta_{q}$ as well as the observer gain $L$. Packet losses are caused by the disturbance in communication channels. Therefore $P_{l}$ is influenced by the working environment and usually can not be designed. The quantization parameter $\delta_{q}$ is obviously determined by the applied quantizer in source coding. A lager $\delta_{q}$ implies a smaller quantization density $\rho$, that means a coarser quantizer can be applied. The observer gain $L$ is a free design parameter. Hence we formulate the fault detection system design as three optimization problems:

Problem 1: Design of the digital channel. For a given observer gain $L$, if the packet loss probability $P_{l}$ is known, find the maximum allowable $\delta_{q}$ such that

$$
\frac{\|r\|_{2}}{\|d\|_{2}}<\mu
$$


where $\mu>0$ is a given constant.

Problem 2: Design of the FDF. For given communication channel, i.e. $P_{l}$ and $\delta_{q}$ are known, find an observer gain $L$ such that

$$
\frac{\|r\|_{2}}{\|d\|_{2}} \rightarrow \min
$$

is fulfilled.

Problem 3: Integrated design of the digital channel and FDF. Given $\mu>0$ and $P_{l}$, find an observer gain $L$ and $\delta_{q}$ such that (7) is satisfied.

The basic idea of the optimization problems is to design the channel parameters and/or observer gain, such that the influence of disturbances on the fault detection system is bounded or minimized. All three problems can be solved with the help of Theorem 1 .

In Problem 1 the maximum allowable $\delta_{q}$ is determined in order to achieve the expected fault detection performance with a given $L$. The following corollary gives the result.

Corollary 1: Given the process (2), FDF (3) with a known $L$, and a constant $\mu>0$, if the packet loss probability $P_{l}$ is known, the maximum allowable $\delta_{q}$ satisfying (7) can be obtained by solving the following optimization problem

$$
\max _{Y>0, \varepsilon>0} \delta_{q}
$$

subject to inequality (6) with

$$
N_{j}=\left[\begin{array}{cc}
A_{j} & E_{d, j} \\
C_{j} & F_{d, j}
\end{array}\right], R_{j}=\left[\begin{array}{c}
E_{j} \\
F_{j}
\end{array}\right], j=0, \cdots, l .
$$

In Problem 2 the optimal observer gain $L$ is designed for the fault detection over a given channel.

Corollary 2: Given the process (2), FDF (3), packet loss probability $P_{l}$, and quantization parameter $\delta_{q}$, the optimal observer gain $L$ satisfying (8) can be selected by solving

$$
\min _{Y_{1}>0, Y_{2}>0, X_{2}} \mu^{2}
$$

with $\mu>0$ subject to

$$
\left[\begin{array}{cccccc}
-S & 0 & \Pi_{13} & \Pi_{14} & \Pi_{15} & 0 \\
* & -\frac{1}{\sigma_{l}^{2}} S & \Pi_{23} & \Pi_{24} & \Pi_{25} & 0 \\
* & * & Y & 0 & 0 & G^{T} \\
* & * & * & \mu^{2} \varepsilon I & 0 & J^{T} \\
* & * & * & * & -I & 0 \\
* & * & * & * & * & -I
\end{array}\right]<0
$$

with

$$
\begin{aligned}
& S=\left[\begin{array}{cc}
Y & 0 \\
0 & \varepsilon I
\end{array}\right], Y=\left[\begin{array}{cc}
Y_{1} & 0 \\
0 & Y_{2}
\end{array}\right] \\
& \Pi_{13}=\left[\begin{array}{cc}
Y_{1} A_{c l} & 0 \\
0 & Y_{2} A_{c l}-\left(1-P_{l}\right) X_{2} C_{c l} \\
0 & \varepsilon\left(1-P_{l}\right) C_{c l}
\end{array}\right] \text {, } \\
& \Pi_{14}=\left[\begin{array}{c}
Y_{1} E_{d, c l} \\
Y_{2} E_{d, c l}-\left(1-P_{l}\right) X_{2} F_{d, c l} \\
\varepsilon\left(1-P_{l}\right) F_{d, c l}
\end{array}\right] \text {, } \\
& \Pi_{15}=\left[\begin{array}{cc}
0 & 0 \\
\left(1-P_{l}\right) \delta_{q} X_{2} & 0 \\
\varepsilon\left(1-P_{l}\right) \delta_{q} & 0
\end{array}\right]
\end{aligned}
$$

$$
\begin{gathered}
\Pi_{23}=\left[\begin{array}{cc}
0 & 0 \\
0 & -X_{2} C_{c l} \\
0 & \varepsilon C_{c l}
\end{array}\right], \\
\Pi_{24}=\left[\begin{array}{c}
0 \\
-X_{2} F_{d, c l} \\
\varepsilon F_{d, c l}
\end{array}\right], \\
\Pi_{25}=\left[\begin{array}{cc}
0 & 0 \\
\delta_{q} X_{2} & 0 \\
\varepsilon \delta_{q} & 0
\end{array}\right]
\end{gathered}
$$

and $L=Y_{2}^{-1} X_{2}$.

In Problem 3 the observer is designed such that the requirements on QoS of digital channels for an expected FD performance are minimized, that means the acceptable uncertainty introduced by the quantization is maximized. This problem can be solved in the similar way as in Problem 2.

Corollary 3: Given the process (2), FDF (3) and a constant $\mu>0$, the observer gain $L$ and maximum allowable $\delta_{q}$ solving Problem 3 can be determined by

$$
\max _{Y_{1}>0, Y_{2}>0, X_{2}} \delta_{q}
$$

subject to the inequality (9) and $L=Y_{2}^{-1} X_{2}$.

In this case (9) is no longer a linear matrix inequality, but it can be solved by using iterative methods.

\section{Residual evaluation}

Since an evaluation of residual signals over the whole time is usually unrealistic, the evaluation function in this paper is computed in a time window, which is

$$
\|r\|_{t}=\left[\sum_{i=k-t}^{k} r(i)^{\prime} r(i)\right]^{1 / 2}
$$

where $t=1,2, \cdots$ is an integer denoting the length of the evaluation window.

In case of no fault, the residual is determined by $d(k)$ and the threshold can be chosen as $J_{t h}=\mu \delta_{d}$, where $\|d\|_{2} \leq \delta_{d}$. Then the occurrence of faults can be tested according to the following logic rule

$$
\begin{aligned}
\|r\|_{t} & >J_{t h} \Rightarrow \text { fault alarm } \\
\|r\|_{t} & \leq J_{t h} \Rightarrow \text { fault-free } \\
& \text { IV. EXAMPLE }
\end{aligned}
$$

To illustrate the results, we take the following dynamic process as an example:

$$
\begin{aligned}
& A=\left[\begin{array}{ll}
2 & 1 \\
0 & 1
\end{array}\right], B=\left[\begin{array}{ll}
1 & 0 \\
0 & 1
\end{array}\right], C=\left[\begin{array}{ll}
1 & 0 \\
0 & 1
\end{array}\right], \\
& E_{d}=\left[\begin{array}{l}
0.1 \\
0.1
\end{array}\right], F_{d}=0, \\
& E_{f}=\left[\begin{array}{l}
1 \\
0
\end{array}\right], F_{f}=0, \\
& K=\left[\begin{array}{cc}
-1.5 & 0 \\
0 & -0.5
\end{array}\right] .
\end{aligned}
$$


Here a static output feedback controller is applied in the process. The sampling rate of the system is $0.1 s$. The norm of disturbance $\|d\|_{2}$ is bounded by 2 and the packet loss probability of the given channel is assumed to 0.1. An actuator fault is generated at $t=250 \mathrm{~s}$ with $f=0.15$. The length of evaluation time window is 100 time steps.

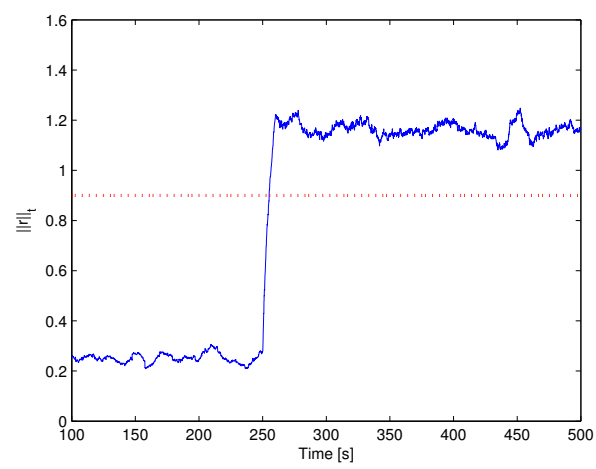

Fig. 2. Results of Problem 1: Evaluated residual signal $\|r\|_{t}$ (solid line) and $J_{t h}=0.9$ (dash line).

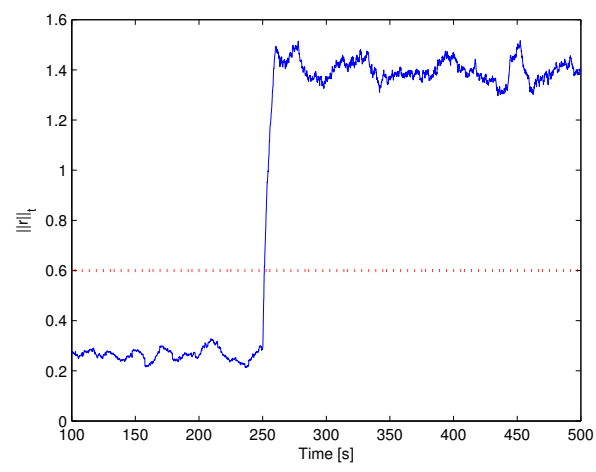

Fig. 3. Results of Problem 2: Evaluated residual signal $\|r\|_{t}$ (solid line) and $J_{t h}=0.6$ (dash line).

For Problem 1, with $\mu^{2}=0.2$ and a given observer gain

$$
L=\left[\begin{array}{cc}
0.4 & 1 \\
0 & 0.3
\end{array}\right]
$$

we obtain the maximum allowable $\delta_{q}=0.2$ according to Corollary 1 , which means the quantizer used in the source encoder should have $\rho>0.67$. The threshold can be set as $J_{t h}=0.9$.

For Problem 2, with $\delta_{q}=0.2$, by applying Corollary 2 we can get the optimal observer gain

$$
L=\left[\begin{array}{cc}
0.29 & 0.73 \\
-0.1394 & 0.433
\end{array}\right]
$$

with $\mu^{2}=0.09$. Then the threshold can be set as $J_{t h}=0.6$.

For Problem 3, with $\mu^{2}=0.1$, according to Corollary 3 the optimal observer gain is

$$
L=\left[\begin{array}{cc}
-0.0639 & -0.0181 \\
0.0086 & -0.0127
\end{array}\right]
$$

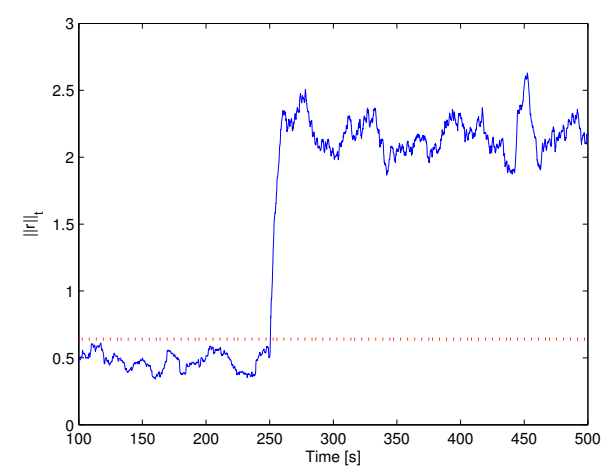

Fig. 4. Results of Problem 3: Evaluated residual signal $\|r\|_{t}$ (solid line) and $J_{t h}=0.63$ (dash line).

and the maximum allowable $\delta_{q}$ is 0.3 . The quantizer will require $\rho>0.54$, and thus a coarser quantizer can be applied here. The threshold is set as $J_{t h}=0.63$.

Fig. 2, 3 and 4 show the simulation results. For different design problems, the maximum allowable $\delta_{q}$ and/or an optimal observer gain $L$ can be designed. With the computed thresholds, the faults can be detected in time.

\section{CONCLUSION}

In this paper an observer-based fault detection system over unreliable digital channels was designed. The QoS, including quantization error and packet loss probability, were analyzed and modeled as stochastic variables and system uncertainties. Then the system was described in the framework of stochastic uncertain systems, where the digital channel and FDF were considered as a whole system. Based on this description, three optimization problems for fault detection were formulated. By solving those problems, the digital channels and FDF were designed. An integrated design approach of control part and communication part in NCS was also proposed. At the end the residual signals were evaluated and compared with a threshold in order to detect the faults.

\section{APPENDIX}

Proof outline of Theorem 1. Let

$$
V(x(k))=x^{T}(k) P x(k), P>0 .
$$

It is evident that

$$
\begin{aligned}
E(V(x(k+1)))-E(V(x(k))) & +E\left(r^{T}(k) r(k)\right) \\
& \left.-\mu^{2} w^{T}(k) w(k)<Q 10\right)
\end{aligned}
$$

with $\mu>0$ ensures

$$
\sum_{k=0}^{\infty} E\left(r^{T}(k) r(k)\right)-\mu^{2} w^{T}(k) w(k)<0 .
$$

The inequality (10) is equivalent with

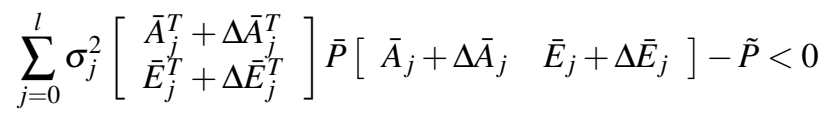


where

$$
\begin{aligned}
\bar{A}^{T} & =\left[\begin{array}{ll}
A_{0}^{T} & C_{0}^{T}
\end{array}\right], \Delta \bar{A}^{T}=\left[\begin{array}{ll}
\Delta A^{T} & \Delta C^{T}
\end{array}\right], \\
\bar{E}^{T} & =\left[\begin{array}{ll}
E_{w, 0}^{T} & F_{w, 0}^{T}
\end{array}\right], \Delta \bar{E}^{T}=\left[\begin{array}{ll}
\Delta E_{w}^{T} & \Delta F_{w}^{T}
\end{array}\right]
\end{aligned}
$$

and

$$
\bar{P}=\operatorname{diag}(P, I), \tilde{P}=\operatorname{diag}\left(P, \mu^{2} I\right), \sigma_{0}^{2}=1 .
$$

Define

$$
P_{j}=\sigma_{j}^{2}\left[\begin{array}{ll}
P & 0 \\
0 & I
\end{array}\right]
$$

and

$$
\begin{gathered}
M_{j}=\left[\begin{array}{cc}
A_{j}+\Delta A_{j} & E_{w, j}+\Delta E_{w, j} \\
C_{j}+\Delta C_{j} & F_{w, j}+\Delta F_{w, j}
\end{array}\right], N_{j}=\left[\begin{array}{cc}
A_{j} & E_{w, j} \\
C_{j} & F_{w, j}
\end{array}\right], \\
j=0, \cdots, l
\end{gathered}
$$

then (10) can be written as

$$
\begin{gathered}
{\left[\begin{array}{lll}
M_{0}^{T} & \cdots & M_{l}^{T}
\end{array}\right]\left[\begin{array}{ccc}
P_{0} & & 0 \\
& \ddots & \\
0 & & P_{l}
\end{array}\right]\left[\begin{array}{c}
M_{0} \\
\vdots \\
M_{l}
\end{array}\right]} \\
-\left[\begin{array}{cc}
P & 0 \\
0 & \mu^{2} I
\end{array}\right]<0
\end{gathered}
$$

Applying Schur-complement yields

$$
\left[\begin{array}{cccc}
-P_{0}^{-1} & & \multicolumn{2}{c}{M_{0}} \\
& \ddots & & \\
& & -P_{l}^{-1} & M_{l} \\
M_{0}^{T} & \cdots & M_{p}^{T} & {\left[\begin{array}{cc}
-P & 0 \\
0 & -\mu^{2} I
\end{array}\right]}
\end{array}\right]<0
$$

which is equivalent with

$$
\begin{aligned}
& {\left[\begin{array}{cccc}
-P_{0}^{-1} & & \multicolumn{2}{c}{N_{0}} \\
& \ddots & & \\
& & -P_{l}^{-1} & N_{l} \\
N_{0}^{T} & \cdots & N_{p}^{T} & {\left[\begin{array}{cc}
-P & 0 \\
0 & -\mu^{2} I
\end{array}\right]}
\end{array}\right]+} \\
& {\left[\begin{array}{c}
E_{0} \\
F_{0} \\
\vdots \\
E_{l} \\
F_{l} \\
0
\end{array}\right] \Delta\left[\begin{array}{c}
0_{1} \\
\vdots \\
0_{l} \\
G^{T} \\
J^{T}
\end{array}\right]^{T}+\left[\begin{array}{c}
0_{1} \\
\vdots \\
0_{l} \\
G^{T} \\
J^{T}
\end{array}\right] \Delta^{T}\left[\begin{array}{c}
E_{0} \\
F_{0} \\
\vdots \\
E_{l} \\
F_{l} \\
0
\end{array}\right]^{T}<0}
\end{aligned}
$$

Here we introduce a useful lemma to deal with uncertainties.

Lemma 1: [13] [15] Given the matrices $\tilde{H}$ and $\tilde{E}$ of appropriate dimensions, then

$$
\tilde{H} \Delta \tilde{E}+\tilde{E}^{T} \Delta^{T} \tilde{H}^{T}<0
$$

where $\Delta$ is as in (5), if and only if there exits a scalar $\varepsilon>0$ such that

$$
\left[\begin{array}{cc}
\varepsilon^{-1 / 2} \tilde{E}^{T} & \varepsilon^{1 / 2} \tilde{H}
\end{array}\right]\left[\begin{array}{c}
\varepsilon^{-1 / 2} \tilde{E} \\
\varepsilon^{1 / 2} \tilde{H}^{T}
\end{array}\right]<0
$$

Applying the lemma, we get

$$
\begin{gathered}
{\left[\begin{array}{cccc}
-P_{0}^{-1} & \multicolumn{2}{c}{N_{0}} \\
& \ddots & \\
& \multicolumn{2}{c}{P_{l}^{-1}} & \\
N_{0}^{T} & \ldots & N_{p}^{T} & {\left[\begin{array}{cc}
-P & 0 \\
0 & -\mu^{2} I
\end{array}\right]}
\end{array}\right]+} \\
\varepsilon\left[\begin{array}{c}
E_{0} \\
F_{0} \\
\vdots \\
E_{l} \\
F_{l} \\
0
\end{array}\right]\left[\begin{array}{c}
E_{0} \\
F_{0} \\
\vdots \\
E_{l} \\
F_{l} \\
0
\end{array}\right]^{T}+\frac{1}{\varepsilon}\left[\begin{array}{c}
0_{1} \\
\vdots \\
0_{l} \\
G \\
J
\end{array}\right]\left[\begin{array}{c}
0_{1} \\
\vdots \\
0 \\
G \\
J
\end{array}\right]<0
\end{gathered}
$$

which implies (10). By applying Schur-complement and similarity transformation, finally we obtain the linear matrix inequality (6) with $Y=\varepsilon P$.

\section{REFERENCES}

[1] R. W. Brockett and D. Liberzon, "Quantized feedback stabilization of linear systems", IEEE Trans. Automat. Contr., vol 45, pp 1279-1289, 2000.

[2] N. Elia and S. Mitter, "Stabilization of linear systems with limited information", IEEE Trans. Automat. Contr., vol 46, pp 1384-1400, 2001.

[3] Z. Gao, H. Wang, T. Chai, "A robust fault detection filtering for stochastic distribution systems via descriptor estimator and parametric gain design", IET Proc. - Control Theory Applications, vol.1, no.5, pp.1286-1293, 2007.

[4] Z. Gao and D. W. C. Ho, "State/noise estimator for descriptor systems with application to sensor fault diagnosis", IEEE Transactions on Signal Processing, vol.54, no.4, pp.1316-1326, 2006.

[5] H. Haimovich, M. M. Seron and G. C. Goodwin, "Geometric characterization of multivariable quadratically stabilizing quantizer", Int. J. Control, vol 79, pp 845-857, 2006.

[6] H. Ishii and T. Basar, "Remote control of LTI systems over networks with state quantization", System \& Control Letters, vol 54, pp 15-31, 2005.

[7] O. C. Imer, S. Yksel and T. Basar, "Optimal control of LTI systems over unreliable communication links", Automatica, vol 42, pp 14291439, 2006.

[8] Z. Jin, V. Gupta and R. M. Murray, "State estimation over packet dropping networks using multiple description coding", Automatica, vol 42, pp 1441-1452, 2006.

[9] D. Liberzon, "Hybrid feedback stabilization of systems with quantized signals", Automatica, vol 39, pp 1543-1554, 2003.

[10] P. Seiler and R. Sengupta, "An $H_{\infty}$ approach to networked control", IEEE Trans. Automat. Contr., vol. 50, pp 356-364, 2005.

[11] B. Sinopoli, L. Schenato, M. Franceschetti, K. Poolla, M. I. and S. S. Sastry, "Kalman filtering with intermittent observations", IEEE Trans. Automat. Contr., vol. 49, pp 1453-1464, 2004.

[12] J. Wu and T. Chen, "Design of networked control systems with packet dropouts", IEEE Trans. Automat. Contr., vol. 52, pp 1314-1319, 2007

[13] L. Xie, "Output feedback $H_{\infty}$ control of systems with parameter uncertainty", Int. J. of Control, vol. 63, pp 741-750, 1996.

[14] J. Xiong and J. Lam, "Stabilization of linear systems over networks with bounded packet loss"', Automatica, vol. 43, pp 80-87, 2007

[15] L. Yu and F. Gao, "Output feedback guaranteed cost control for uncertain discrete-time systems using linear matrix inequalities", $J$. of Optimization theory and applications, vol. 113, pp 621-634, 2002.

[16] P. Zhang, S. X. Ding, P. M. Frank and M. Sader, "Fault detection of networked control systems with missing measurements", 5th Asian Control Conference, Melbourne, Australia, 2004. 